\title{
Evaluating the value of synchrotron radiation phase-contrast CT imaging in the study of knee joint microstructure
}

\author{
Meibai Tang ${ }^{1}$, Richeng Zhi ${ }^{1}$, Changyi Sun ${ }^{1}$, Xiaohua Wang ${ }^{1}$, Chenglin Liu ${ }^{1 *}$ \\ ${ }^{1}$ School of Physics and Electronic Engineering, Yancheng Teachers University, Yancheng 224007, China
}

\begin{abstract}
The knee joint is one of the tissues that often appear diseased, and the pathogenesis owing to its structure. Therefore, the micro-structure of the knee joint was studied by synchrotron radiation phasecontrast CT imaging (SRPCI-CT). The contrast and the sharpness of the reconstructed slice were calculated and analysed. From these results, the layer structure of the joint tissue can be seen. The cartilage layer (including the meniscus) is about $1.44 \mathrm{~mm}$, and the epiphyseal layer is about $2.25 \mathrm{~mm}$, which conforms to the actual situation of rat knees. From the 3D images, the structure of the meniscus and the epiphyseal bone can be observed. The epiphyseal bone line is also particularly obvious. Simultaneously, the epiphyseal bone also has a mesh structure, which has the characteristics of both cartilage and tibia. Further research on the tibia can determine that the trabecular bone line is about 130 microns. Finally, the bone volume fraction (BVF) of the joint, the data also reflect the existence of a layer structure, and its scale distribution is consistent with the results of sharpness and contrast analysis. The SRPCI-CT can play an important role in the study micro-structure of joints.
\end{abstract}

\section{Introduction}

The knee joint is the largest and most complex tissue in the human body. Its anatomical structure is that the upper is the femur and the lower is the tibia. There is an articular surface between the two bones [1]. A layer of cartilage on the surface of the joint is approximately a few millimeters thick [2]. There are cruciate ligaments anteriorly and posteriorly, collateral ligaments on the medial and lateral sides, with a meniscus sandwiched between them. This kind of structure makes sure that the joints are flexible and stable [3]. Knee arthritis is the most common skeletal muscle disease and the main cause of disability in the elderly [4-5]. Therefore, it is necessary to study thoroughly the microstructure of the knee joint.

In the fields of biology and medical science, X-ray imaging is the most important diagnostic tool. In the past 100 years, the traditional X-ray imaging technology based on absorption contrast, which has been extremely commonly used in clinical medicine [6-9]. In absorption imaging, the imaging of low-contrast details of soft tissue has not yielded satisfactory results, and mammography is a typical traditional X-ray imaging method. X-ray phase contrast imaging (PCI) not only uses the absorption contrast, but also uses the refraction contrast. This method is imaged by the refraction effect produced by the difference in density. X-ray phase contrast imaging is a forceful tool for studying weak absorbing materials. The advantages of PCI method are very obvious for studying soft tissues [10].
Because the joint surface is a special tissue structure, its density is between bone and soft tissue, the absorption coefficient of X-rays is basically the same, and it is difficult to distinguish only using the absorption image (such as mammography), but the phase contrast caused by the density change is relatively strong [11-12]. The PCI imaging method can record the phase contrast, then the clarity of the image is relatively high, and in human soft tissue, the density of the meniscus and the surrounding bone tissue due to the density is different, and phase contrast imaging can achieve satisfactory results. Thence, the phase contrast imaging method has grown up to be a very effective method for joint disease diagnosis [13].

$\mathrm{X}$-ray phase contrast imaging has usually different methods, in-line holographic imaging is one of the common methods [12]. Combination of phase contrast imaging and computed tomography (PCI-CT) has been widely used. PCI-CT technology is belonged to direct imaging through the edge enhancement of samples. This method is also suit to make microscopic or CT imaging, such as microscopic tomography ( -CT) [13]. The CT can be used in researching the micro-structure of biological tissue. Because of its high resolution, it has gradually become one of the most effective methods in studying the mechanism of lesions [14-16]. The advantages of synchrotron radiation X-ray intensity, and coherence make this method as a useful tool for biomedical research [17-18]. Therefore, synchrotron radiation micro-imaging technology can provide a significant means to research joint microstructure.

\footnotetext{
* Corresponding author: 1clyctc@163.com
} 
In this paper, the projected images of the knee joints were studied with synchrotron radiation -based phase contrast CT imaging (SRPCI-CT). These images were reconstructed after phase recovery. The hierarchical structure of the knee joint was analyzed according to the sharpness and contrast of the reconstructed slice and the bone volume fraction of the $3 \mathrm{D}$ image. The role of SRPCI-CT in the joint layer structure analysis was evaluated.

\section{Experimental samples and Methods}

\subsection{The experimental sample processing}

The experimental animals are three months' old sprague dawley (SD) rats. These SD rats are provided by the Shanghai laboratory animal research center, Chinese academy of sciences (manufacturing license No. SCXK (Shanghai) 2012-0002). The rats were anesthetized with a large dose of anesthetic. The abdominal aorta is injected with $5 \%$ sodium citrate solution to prevent blood clotting, to draw blood and clear blood vessels. At last, the contrast agent (hundreds of nanometers of microfil perfusion agent) as was injected into the blood vessels. After 15 minutes, the hind limb of rats was taken, then soaked in $10 \%$ formalin solution for the experimental sample. The shape of the knee joint sample is similar to the $10 \mathrm{~mm}$ height, $7 \mathrm{~mm}$ diameter cylinder. The sample was placed in the cylindrical plastic pipe in the PCI-CT imaging process.

\subsection{The collection of projection images}

The in-line PCI-CT projection images of the experimental samples were recognized in BL13W1 station, SSRF (Shanghai Synchrotron Radiation Facility) [19-20]. The basic parameters of the imaging process are the monochromatic light energy was $20 \mathrm{keV}$, the spot on the sample was approximately $29,100 \mathrm{~mm} \times 5,229 \mathrm{~mm}$, the size of the image was $2,900 \times 581$ pixels (each pixel size with $9 \mathrm{~mm}$ ), the distance from the sample to the detector was $0.5 \mathrm{~m}$, the exposure time was $5 \mathrm{~ms}$, the objective table was rotated $180^{\circ}$ with $0.2^{\circ} / \mathrm{s}$ scanning speed.

\subsection{The phase information extracted and 3D reconstruction}

The projection image were extracted phase information using phase-attenuation duality Paganin algorithm (PAD-PA) in PITRE software [21-22]. The slice was also reconstructed with PITRE software. The threedimensional images were reconstructed by Amira software. At the same time, the clarity, contrast and bone volume fraction are calculated from slice and 3D images.

\section{Results}

\subsection{The projected image and phase information extraction}

Fig. 1 is the projected images of the knee joint recognized by the PCI-CT method. These images start from the initial position and correspond to the projection images taken every 20 degrees. Figure 1(A) coincides with the initial position ( 0 degrees), and figure $1(\mathrm{~J})$ corresponds to the last position (180 degrees). When actually collecting the projected image, record an image every 0.2 degrees, a total of 900 images. These images contain the absorption and small-angle scattering (extinction) information, and reduce the image quality. The image's contrast can be improved through virtue of the extinction effect.

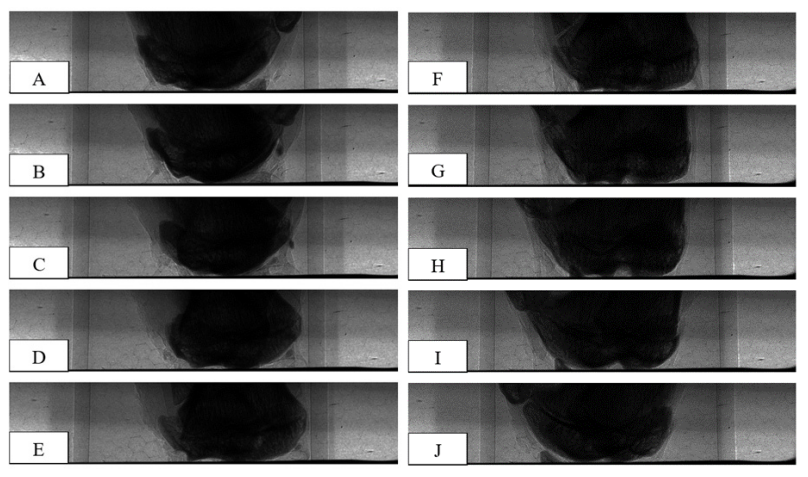

Fig. 1. The projection images of knee joint. These images start from the initial position (Fig.1 (A), corresponds to 0 degrees), each picture is separated by 20 degrees, and Fig. 1(J)

corresponds to 180 degrees (that is, the last picture). When actually collecting the projected image, record an image every 0.2 degrees, a total of 900 images.

To better display the microstructure contained in the projected image, which needs to phase extraction. The image after phase recovery (referred to as phase recovery image) is shown in Fig. 2. In the information extraction process, the structure is almost the same between the algorithm image and the original image. However, in the algorithm image, the meniscus, epiphyseal bone, tibia, and fibula have become obvious, and some microstructures are also revealed, especially the epiphyseal bone line is very obvious.

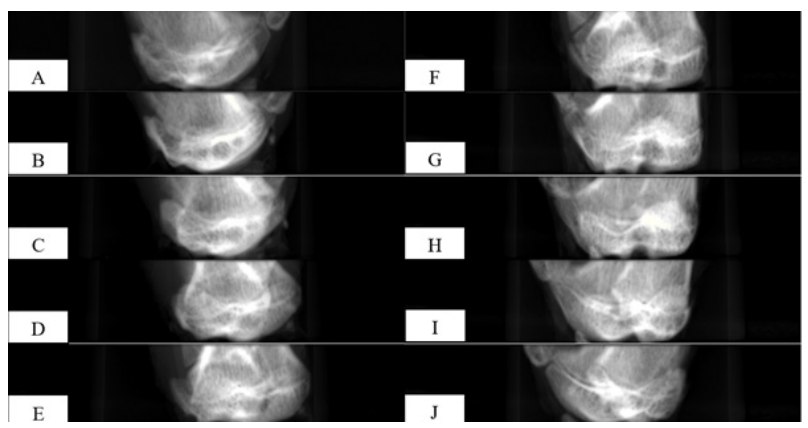

Fig. 2. The phase recovery image after obtaining by PAD-PA algorithm. The angular positions corresponding to $\mathrm{A}$ to $\mathrm{J}$ are consistent with Fig. 1. 


\subsection{The reconstructed slice on the images after phase recovery}

The slices are reconstructed images using a large number of phase recovery images, which is also the structure image of the experimental samples. Fig. 3 is the slice images reconstructed after processed by PAD-PA algorithm. Fig. 3(A) is a slice of nearly knee joint cavity, which corresponds to slice number 580. Fig. 3(F) is the slice of backwards to the tibia and fibula, corresponds to slice number 100 , can view the prototype of a separate tibia and fibula. In Fig. 3(B), you can see the two parts of the meniscus, the front and back, contain the cruciate ligament. It can also show that the microstructure of the meniscus is significantly different from the surrounding soft tissue (muscle). Fig. 3(C) shows that the discrete structure of the meniscus has formed a whole structure, that is, the structure of the epiphyseal bone, and the structural proportion of its bone has increased significantly. Fig. 3(D) is a structure of epiphyseal differentiation. It is in the transitional stage of differentiation of epiphyseal bone to the tibia and fibula. The presence of an epiphyseal bone lines between the epiphyseal bone and tibia makes the figure present a separate structure. Fig. 3(E) shows the obvious microstructure of the tibia and fibula, and highlights the separation between the tibia and the fibula.

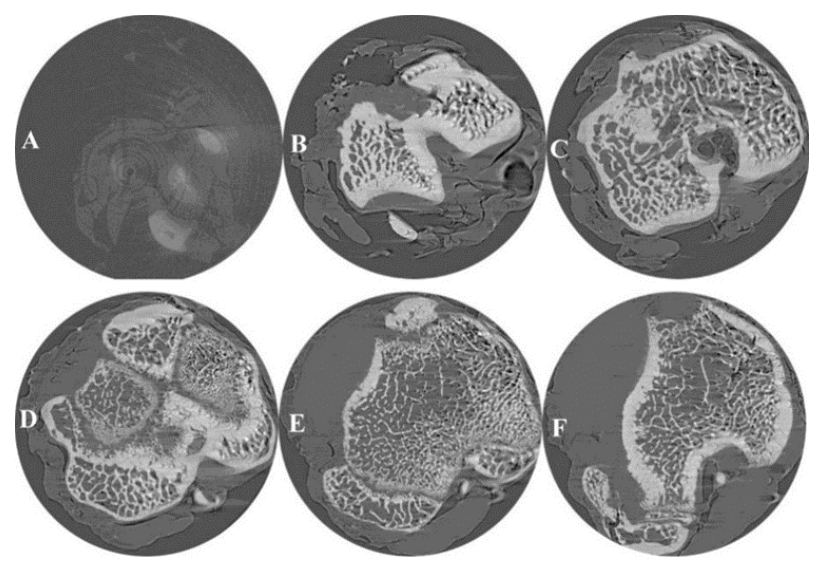

Fig. 3. The reconstructed slice using the projection images.

A - First fault image (near knee joint cavity) B-slice

No.500 C-slice No.400 D-slice No.300 E-slice

No.200 F-slice No.100 (backwards to the tibia and fibula)

\subsection{D images}

For better analysing the internal structure of bone, the internal part of the knee joint sample was captured, then reconstructed using three-dimensional (3D) CT images. These images are shown in Fig. 4. The honeycomb structure of the joint can be indeed seen from the 3D image, especially can well show the internal structure of the tibia. From the top down (Fig. 4(A)), the shape of the meniscus and cruciate ligament on the articular surface can be clearly seen in the picture. From the side of Fig. 4(B), you can see the meniscus, epiphysis, tibia, and fibula, especially the epiphyseal line between the tibia and the epiphysis. The two ends of the bone are mostly epiphysis, and the articular surface is the subject of smooth articular cartilage. The epiphyseal line of the junction of the epiphysis and the backbone is more obvious. The epiphyseal line is a layer of cartilage, which is the ossification point that appears in the cartilage at both ends of the bone during the development process. Similarly, the uneven distribution of the density of the epiphyseal bone can be seen. Through the proliferation and ossification of chondrocytes, the bone is continuously lengthened.

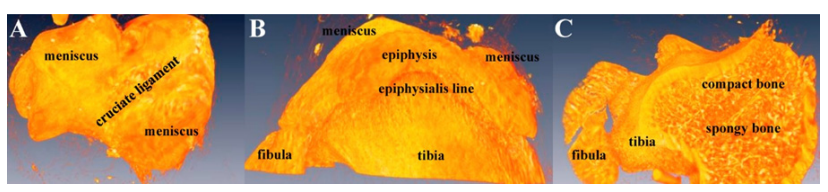

Fig. 4. The 3D images of samples by PCI-CT imaging

$\mathrm{A}$ - superior aspect $\mathrm{B}$ - front aspect $\mathrm{C}$-inferior aspect

In the bottom view of the bone tissue (Fig. 4(C)), the structure of the tibia and fibula can be seen, especially the difference between the dense and cancellous bone in the tibia. There is a plentiful internal network structure near the knee, and the honeycomb structure is good, reflecting the good bearing capacity. In the tibia, there is only an obvious network structure, which contains a large number of trabecular bones.

\section{Discussion}

\subsection{The distribution of trabecular size}

Fig. 5 is the distribution of trabecular size in the tibia, taken as an example using slice No. 400 (corresponds to Fig. 3 (C)). It can display not only a structural situation of bone and bone marrow in Fig. 5, but also the diameter and distribution of vascular and trabecular bone in all kinds of slices. Adopt a typical picture (slice No. 400) to analyze it. The corresponding gray scale changes, there are 16 different distinct peaks in the range of about 6.5 $\mathrm{mm}$ (shown in Fig. 6). According to the half-height width of the curve, the largest trabecular bone size is 216 microns. The smallest size is 63 microns, and the average size is 129.3646 .11 microns.

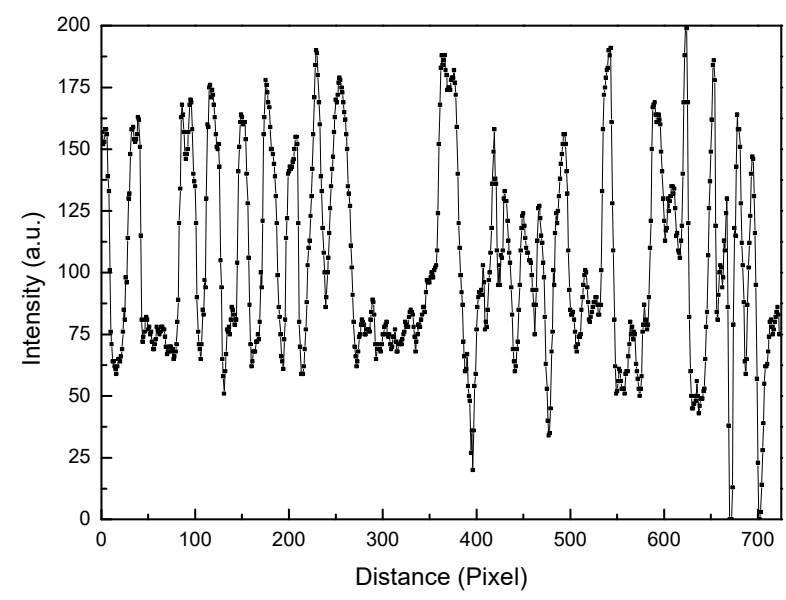

Fig. 5. The distribution of trabecular size in tibia, take as an example using slice No. 400 (corresponds to Figure 3 (C)) 


\subsection{The distribution of the contrast and the sharpness}

We analyze the sharpness and contrast of the slice in order to discuss the quality of the reconstructed image [23-24]. Comparing their performance used the contrast of the image. The small difference of the contrast represents the harder distinguishing image. The contrast of the reconstructed slice are displayed in Fig. 6 (black line). Similarly, the sharpness of the reconstructed slice is shown in Fig. 6 (red line). The contrast and the sharpness are roughly divided into three areas in Fig.6. The area between slices No. 580 and 420 should be the cartilage corresponding to the joint, with an approximate thickness of about $1.44 \mathrm{~mm}$. Since articular cartilage has both the characteristics of bone and soft tissue, the contrast is relatively high, but as the composition of bone decreases, the density of soft tissue increases, and the contrast decreases sharply. The second area is in the range of slice No. 420-170, and the approximate thickness is $2.25 \mathrm{~mm}$. It is that one where the articular cartilage and tibia are tightly closed, which is an important part of joint support. In this area, the peak change in contrast, which may be related to the reticular structure of the tibia bone tissue. In the area after slice No. 170, the tibia and fibula can be surely seen, the absorption contrast is unchanged, and it also reflects the characteristics of traditional medical imaging.

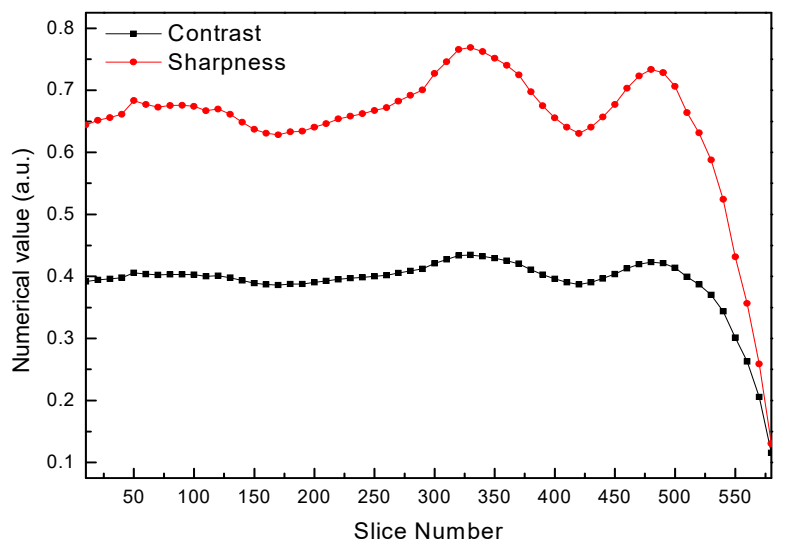

Fig. 6. The contrast (black line) and the sharpness (red line) of the reconstructed slice.

\subsection{The distribution of the bone volume fraction}

The bone volume fraction (BVF) was calculated in 3D images [25]. The results of BVF are shown in Fig. 7. The distribution of the BVF is consistent with the characteristics reflected by the image sharpness and contrast. The section 420-580 should be in the area of the articular surface (including the epiphysis and the meniscus). The volume is quite low, mainly because cartilage is the main area in this area. If the calcification is not high, the bone composition is not rich. The second segment is in the range of $170-420$, this segment corresponds to the dense area of the tibia, the bone is dense in texture, strong in compression and distortion, and is made available on the surface of the bone. The third segment is after slice number 170 , this segment is still dominated by the tibia, but corresponds to the cancellous area of the tibia, and the bone cancellous is constituted of lots of bone trabeculae intertwined with each other and is spongy. The orientation of the trabecular is in line with the direction of pressure or tension on the bone.

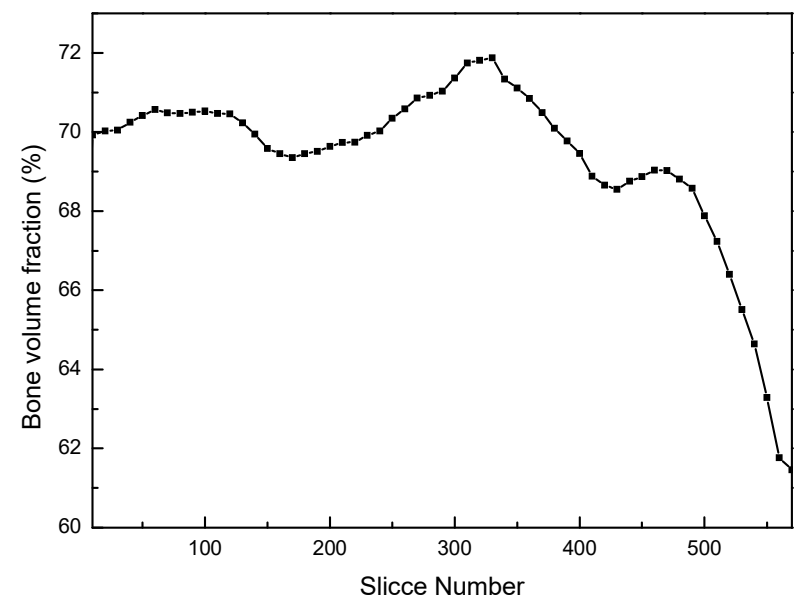

Fig. 7. The bone volume fraction of knee joint.

\section{Conclusions}

The microstructure of the knee joint was studied using the SRPCI- CT in this paper. The phase information of the projection images was recovered using PAD-PA algorithm and reconstructed in PITRE software. The reconstructed three-dimensional images were analysis and comparison. Regardless of the sharpness, contrast, or the volume ratio, the meniscus, epiphysis, and tibia are very distinct in the entire joint area. The meniscus is dominated by soft tissue, and the epiphysis is a spongy tissue mixed with bone structure, while the tibia is obviously seen that its edge is dense and internal bone is cancellous. The size of the trabecular bone inside the tibia is about 100 microns, and it is a network structure. Therefore, the SRPCI-CT imaging can play an important role in the analysis of joint microstructure.

\section{Acknowledgment}

This study received the College Students' Innovative Entrepreneurial Training Plan Program of Jiangsu Province, China (201810324002) and the National Natural Science Foundation of China (No. 11175152).

\section{References}

1. Yim J. H., Seon J K, Song E K, Choi J I, Kim M C, Lee K B, Seo H Y. The American journal of sports medicine, 41(7), 1565-1570 (2013)

2. Zhao Z W, Zhou K, Li Q, Cai C Y. China J Orthop Trauma, 33(3), 234-237 (2020)

3. LaPrade C M, James E W, Engebretsen L, et al. Knee Surg Sports Traumatol Arthrosc, 22(5), 1119-1123 (2014)

4. Eun S S, Lee S H. Knee, 23(4):740-743 (2016) 
5. Roemer $\mathrm{F}$ W, Kwoh $\mathrm{C} \mathrm{K}$, Hannon $\mathrm{M} \mathrm{J}$, et al. European Radiology, 27(1), 404-413 (2017)

6. Xie H L, Deng B, Du G H, et al. Nuclear Science and Techniques, 26(2), 6-21 (2015)

7. Shi S P, Zhang H R, Yin X Z, et al. Journal of Synchrotron Radiation, 26(5), 1742-1750 (2019)

8. Liu C L, Yan X H, Zhang X Y, et al. Phys. Med. Biol., 52, 419-427 (2007)

9. Liu C L, Wang X H, Xu H, et al. J. Electron Spectrosc. Relat. Phenom., 196, 80-84 (2014)

10.Stefanutti E, Sierra A, Miocchi P, et al. Journal of Instrumentation, 13(03), C03027-C03027 (2018)

11.Effendy N M, Khamis M F, Shuid A N. Curr Drug Targets, 14(13), 1542-1551 (2013)

12.Pei Dong, Sylvain Haupert, Bernhard Hesse, Bone, 60, 172-185 (2014)

13.Vasileios Ch. Korfiatis, Simone Tassani, George K. Matsopoulos. Computers in Biology and Medicine, 87, 358-370 (2017)

14.Zhang D M, Yan X H, Zhang X Y, et al. Anal Bioanal Chem., 401, 803-808 (2011)

15.Chen R C, Dreossi D, Mancini L. Synchrotron Rad. 19, 836-845 (2012)

16.Chen R C, Rigon L, Longo R. Opt. Express, 21, 7384-7399 (2013)
17.Mikiyasu Shirai, Daryl O. Schwenke, Hirotsugu Tsuchimochi, et al. Circulation Research. 112, 209. 221 (2013)

18.Lee J, Jang S, Lee S, et al. Sci Rep, 10, 555 (2020)

19.Liu C L, Xu H, Chen C Y. 2011 4th International Conference on Biomedical Engineering \& Informatics (BMEI), 572-574 (2011)

20.Liu C L, Wang X H. 2017 10th International Congress on Image and Signal Processing, BioMedical Engineering and Informatics (CISPBMEI), 1-6 (2017)

21.Chen R C, Rigon L, Longo R. Phys. D Appl. Phys, 44(49), 495401 (2011)

22.Chen R C, Xie H L, Rigon L, et al. Optics Letters. 36, 1719-1721 (2011)

23.Liu C L, Yan X H, Zhang X Y, et al. Nuclear Techniques, 29, 881-884 (2006)

24.Born M, Wolf E, Principles of Optics: Electromagnetic Theory of Propagation, Interference and Diffraction of Light (7th.Ed). The Press of University of Cambridge, Cambridge, England, 298 (1999)

25.Alomari A H, Wille M L, Langton C M. Bone, 107, 145-153 (2018) 